\title{
Free vibration analysis of functionally graded beams using a higher-order shear deformation theory
}

\author{
Zaoui F. Zohra ${ }^{1 *}$, Hanifi H. A. Lemya ${ }^{2}$, Younsi Abderahman ${ }^{3}$, Meradjah Mustapha ${ }^{4}$, Tounsi \\ Abdelouahed $^{5}$, Ouinas Djamel ${ }^{6}$
}

\author{
${ }^{1,6}$ Laboratory of numerical and experimental modeling of the mechanical phenomena, Mechanical \\ Engineering Department, Faculty of sciences and Technology / Ibn Badis University, Mostaganem \\ 27000, Algeria \\ ${ }^{2}$ Laboratoire de Ressources Hydriques et Environnement, Civil Engineering Department, Faculty \\ of Technology/ University of Moulay Tahar, Saïda 20000, Algeria \\ 3,4,5 Material and Hydrology Laboratory, Civil Engineering Department, Faculty of sciences and \\ Technology/ University of Sidi Bel Abbes 22000, Algeria
}

Email: fatima.zaoui@univ-mosta.dz

\begin{abstract}
This paper presents an analytical solution to the free vibration analysis of functionally graded beams by using a refined hyperbolic shear deformation theory in which the stretching effect is included. The modulus of elasticity of beams is assumed to vary according to a power law distribution in terms of the volume fractions of the constituents. Equations of motion are derived from Hamilton's principle and Navier-type analytical solutions for simply supported beams are compared with the existing solutions to verify the validity of the developed theory. Numerical results are obtained to investigate the effects of the power-law index and sideto-thickness ratio on the natural frequencies. It can be concluded that the present theories are not only accurate but also simple in predicting the free vibration responses of FG beams.
\end{abstract}

Keywords: Analytical Modeling, Beam, Functionally Graded Material, Natural Frequencies, Free Vibration.

\section{INTRODUCTION}

Functionally graded materials (FGMs) are a new class of composite materials that has attracted considerable attraction. Typically, FGMs are made from a mixture of metals and ceramics and further characterized by a smooth and continuous change of the mechanical properties from surface to another and thus eliminate the stress concentration at the interface of the layers found in laminated composites. The potential uses of FGMs in engineering applications include aerospace structures, engine combustion chambers, fusion energy devices, engine parts and other engineering structures. In recent years, the static and dynamic analyses of functionally graded (FG) beams have increasingly attracted many researchers.

Based on the Euler-Bernoulli beam theory, the vibration responses of FGM beams have been widely studied by different approaches. Şimşek and Kocatürk [1] studied the dynamic response of an FGM simply supported beam under a concentrated moving harmonic load, in which the effects of the material homogeneity, the velocity of the moving harmonic load, and the excitation frequency on the dynamic responses of the beam were discussed. Yang and Chen [2] analyzed the free vibration and buckling of FGM beams with the presence of open cracks. Li et al. [3] analyzed a small vibration of post-buckled FGM beams with surface-bonded piezoelectric layers in thermal environment by a numerical shooting method based the exact geometrically non-linear theory for axially extensible beams.

In the framework of the first shear deformation theory or the Timoshenko beam theory, Li [4] presented analytical solutions for the static bending and free vibration of FGM Timoshenko and Euler-Bernoulli beams. Huang and Li [5] also studied the free vibration of axially FGMs with nonuniform cross-sections by using the integration technique to transform the differential governing equations into the Fredholm integral equations. Bouremana et al. [6] presented a new first shear deformation theory based on neutral surface position for FGM beams.

Based on higher order shear deformation theories, studies on bending and vibration of FGM beams were performed. Aydogdu and Tashkin [7] studied the free vibration behavior of a simply supported FGM beam based on the first, parabolic, and exponential shear deformation beam theories, respectively, in which natural frequencies were obtained by the Navier type solution method. Şimşek [8] investigated the dynamic responses of functionally graded beams by different beam theories, in which a system of equations of motion was 
derived by Lagrange's equations. Mahi et al. [9] analyzed the free vibration of FGM beams with the temperature dependent material properties. The formulation was derived based on a unified higher order shear deformation theory. The effects of the initial thermal stress on the natural frequencies were also discussed. Thermal effect on wave propagation of functionally graded plates based on neutral surface position was studied by Boukhari et al. [10]. Bourada et al. [11] presented a new simple and refined trigonometric higherorder beam theory for bending and vibration analysis of FG beams with including the thickness stretching effect.

The purpose of this work is to develop a simple and raffined higher-order shear deformation theory for free vibration behavior of beams. The proposed theory contains fewer unknowns and satisfies the zero traction boundary conditions on the top and bottom surfaces of the beam without using any shear correction factors. The displacement fields are chosen based on hyperbolic variation in the inplane displacements through the thickness. Partitioning the transverse displacement into the bending and shear components leads to a reduction in the number of unknowns, and consequently, makes the present theory much more amenable to mathematical implementation. Equations of motion are derived from Hamilton's principle. Closed-form solutions are obtained for a simply supported beam. A good agreement between the present results and the available solutions existing in the literature are found to prove the validity of the proposed theory.

\section{THEORETICAL FORMULATIONS}

\subsection{Material properties}

A functionally graded beam made of length $L$, width $b$ and thickness $h$, with co-ordinate system $(O x y z)$ having the origin $O$ is considered in this study. The beam geometry and the variation of material volume fraction across the beam thickness associated with the power law distribution are shown in figure 1. Based on the rule of mixture, the effective material properties, $\mathrm{P}$, can be written as:

$$
P(z)=P_{m} V_{m}+P_{c} V_{c}
$$

where $P_{m}, P_{c}, V_{m}$ and $V_{c}$ are material properties and the volume fraction of the metal and ceramic respectively with the relation

$V_{m}+V_{c}=1$

According to the power law distribution, the volume fraction of ceramic can be written as

$V_{c}=\left(\frac{z}{h}+\frac{1}{2}\right)^{p}$

where the positive number, $0 \leq p \leq \infty$, is the power law or volume fraction index. The FG beam becomes a fully ceramic beam when $\mathrm{n}$ is set to zero. From the above relationship, the material properties, in terms of Young's modulus and mass density are expressed as

$$
E(z)=E_{m}+\left(E_{c}-E_{m}\right) V_{c}
$$$$
\rho(z)=\rho_{m}+\left(\rho_{c}-\rho_{m}\right) V_{c}
$$

The Poisson's ratio will assume to be constant in our study.

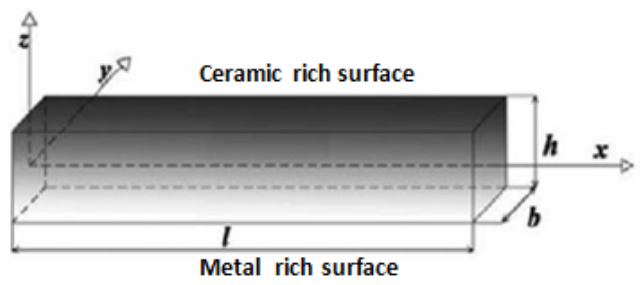

(a)

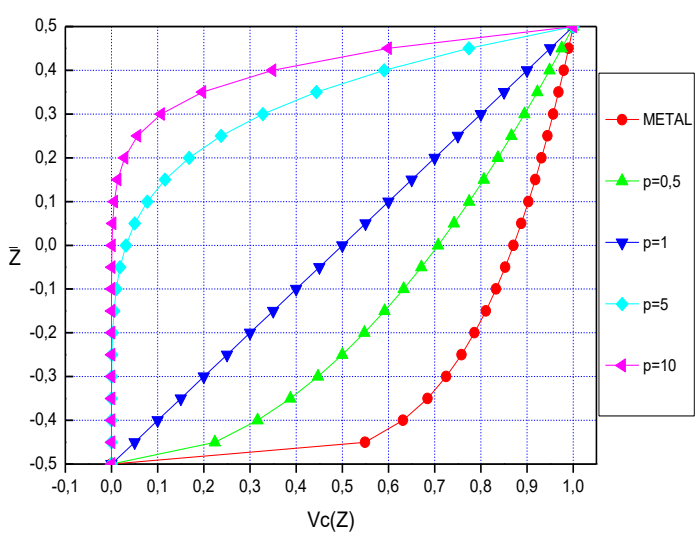

(b)

Figure 1. Geometry of a functionally graded beam and volume fraction profile.

\subsection{Basic assumptions}

The displacement field of the present theory is chosen based on the following assumptions:

$>$ The origin of the Cartesian coordinate system is taken at the neutral surface of the FG beam;

$>$ The transverse displacement is partitioned into bending and thickness stretching components;

$>$ The axial displacement is partitioned into extension, bending and shear components;

$>$ The bending part of the axial displacement is similar to those given by classical beam theory (CBT);

$>\quad$ The shear part of the axial displacement gives rise to the hyperbolic variations of shear strains and hence to shear stresses through the thickness of the beam in such a way that the shear stresses vanish on the top and bottom surfaces of the beam.

\subsection{Kinematics and constitutive equations}

By considering the above hypothesis, the displacement field can be expressed as follow

$$
\begin{aligned}
& u(x, z, t)=u_{0}(x, t)-z \frac{\partial w_{b}}{\partial x}-f(z) \frac{\partial w_{s}}{\partial x} \\
& w(x, z, t)=w_{b}(x, t)+w_{s}(x, t)+g(z) \phi(x, t)
\end{aligned}
$$

In this study, the shape functions $\mathrm{f}(\mathrm{z})$ and $\mathrm{g}(\mathrm{z})$ are chosen based on the hyperbolic function proposed by Zenkour [12]. 
$f(z)=z-h \sinh \left(\frac{z}{h}\right)+\frac{4 z^{3}}{h^{2}} \cosh \left(\frac{1}{2}\right), \quad g(z)=1-f^{\prime}(z)$

The non-zero linear strains derived from Eq. (5) are

$\varepsilon_{x}=\frac{\partial u_{0}}{\partial x}-z \frac{\partial^{2} w_{b}}{\partial x^{2}}-f(z) \frac{\partial^{2} w_{s}}{\partial x^{2}}$

$\varepsilon_{z}=g^{\prime}(z) \varphi(x, t)$

$\gamma_{x z}=g(z)\left(\frac{\partial \varphi}{\partial x}+\frac{\partial w_{s}}{\partial x}\right)$

By assuming that the material of FG beam obeys Hooke's law, the stresses in the beam become

$\sigma_{x}=Q_{11}(z) \varepsilon_{x}+Q_{13}(z) \varepsilon_{z}$

$\sigma_{z}=Q_{13}(z) \varepsilon_{x}+Q_{33}(z) \varepsilon_{z}$

$\tau_{x z}=Q_{55}(z) \gamma_{x z}$

where

$Q_{11}(z)=Q_{33}(z)=\frac{E(z)}{1-v^{2}}, \quad Q_{13}(z)=v Q_{11}(z), \quad$ and $\quad Q_{55}(z)=\frac{E(z)}{2(1+v)}$

\subsection{Equations of motion}

In order to derive the equations of motion, Hamilton's principle is used

$\int_{0}^{T}(\delta U+\delta V-\delta K) d t=0$

where $\delta U, \delta K$ and $\delta V$ denote the strain energy, kinetic energy and the work done by external forces, respectively.

The variation of the strain energy can be stated as

$$
\begin{aligned}
\delta U & =\int_{0}^{L} \int_{-\frac{h}{2}}^{\frac{h}{2}}\left(\sigma_{x} \delta \varepsilon_{x}+\sigma_{z} \delta \varepsilon_{z}+\tau_{x z} \delta \gamma_{x z}\right) d z d x \\
& =\int_{0}^{L}\left(N_{x} \frac{\partial \delta u_{0}}{\partial x}+N_{z} \delta \phi-M_{b} \frac{\partial^{2} \delta w_{b}}{\partial x^{2}}-M_{s} \frac{\partial^{2} \delta w_{s}}{\partial x^{2}}+Q\left(\frac{\partial \delta \phi}{\partial x}+\frac{\partial \delta w_{b}}{\partial x}\right)\right) d x
\end{aligned}
$$

where the stress resultants $N_{x}, M_{b}, M_{s}$ and $Q$ are given by

$$
\begin{aligned}
& \left(N_{x}, M_{b}, M_{s}\right)=\int_{-h / 2}^{h / 2}(1, z, f(z)) \sigma_{x} d z, \\
& N_{z}=\int_{-h / 2}^{h / 2} g^{\prime}(z) \sigma_{z} d z, \\
& Q=\int_{-h / 2}^{h / 2} g(z) \tau_{x z} d z,
\end{aligned}
$$

The variation of work done by externally transverse loads q can be expressed as $\delta V=-\int_{0}^{L} q \delta\left(w_{b}+w_{s}\right) d x$

The variation of the kinetic energy can be expressed as

$$
\begin{aligned}
\delta K & =\int_{0}^{L} \int_{-\frac{h}{2}}^{\frac{h}{2}}[\dot{u} \delta \dot{u}+\dot{w} \delta \dot{w}] \rho(z) d z d x \\
= & \int_{0}^{L}\left\{I_{0}\left[\dot{u}_{0} \delta \dot{u}_{0}+\left(\dot{w}_{b}+\dot{w}_{s}\right)\left(\delta \dot{w}_{b}+\delta \dot{w}_{s}\right)\right]+J_{0}\left(\dot{w}_{b}+\dot{w}_{s}\right) \delta \dot{\phi}\right. \\
& +J_{0} \dot{\phi} \delta\left(\dot{w}_{b}+\dot{w}_{s}\right)-I_{1}\left(\dot{u}_{0} \frac{\partial \delta \dot{w}_{b}}{\partial x}+\frac{\partial \dot{w}_{b}}{\partial x} \delta \dot{u}_{0}\right)+I_{2}\left(\frac{\partial \dot{w}_{b}}{\partial x} \frac{\partial \delta \dot{w}_{b}}{\partial x}\right) \\
& +J_{1}\left(\dot{u}_{0} \frac{\partial \delta \dot{w}_{s}}{\partial x}+\frac{\partial \dot{w}_{s}}{\partial x} \delta \dot{u}_{0}\right)-J_{2}\left(\frac{\partial \dot{w}_{b}}{\partial x} \frac{\partial \delta \dot{w}_{s}}{\partial x}+\frac{\partial \dot{w}_{s}}{\partial x} \frac{\partial \delta \dot{w}_{b}}{\partial x}\right) \\
& \left.+K_{2} \frac{\partial \dot{w}_{s}}{\partial x} \frac{\partial \delta \dot{w}_{s}}{\partial x}+K_{0} \dot{\phi} \delta \dot{\phi}\right\} d x
\end{aligned}
$$

Substituting the expressions for $\delta U, \delta V$, and $\delta K$ from Eqs. (11), (13), and (14) into Eq. (10) and integrating by parts, and collecting the coefficients of $\delta u_{0}, \delta w_{b}, \delta w_{s}$ and $\delta \varphi$. The following equations of motion of the FG beam are obtained

$$
\begin{aligned}
& \delta u_{0}: \frac{\partial N_{x}}{\partial x}=I_{0} \ddot{u}_{0}-I_{1} \frac{\partial \ddot{w}_{b}}{\partial x}-J_{1} \frac{\partial \ddot{w}_{s}}{\partial x} \\
& \delta w_{b}: \frac{\partial^{2} M_{b}}{\partial x^{2}}+q=I_{0}\left(\ddot{w}_{b}+\ddot{w}_{s}\right)+J_{0} \ddot{\phi}+I_{1} \frac{\partial \ddot{u}_{0}}{\partial x}-I_{2} \frac{\partial^{2} \ddot{w}_{b}}{\partial x^{2}}-J_{2} \frac{\partial^{2} \ddot{w}_{s}}{\partial x^{2}} \\
& \delta w_{s}: \frac{\partial^{2} M_{s}}{\partial x^{2}}+\frac{\partial Q}{\partial x}+q=I_{0}\left(\ddot{w}_{b}+\ddot{w}_{s}\right)+J_{0} \ddot{\phi}+J_{1} \frac{\partial \ddot{u}_{0}}{\partial x}-J_{2} \frac{\partial^{2} \ddot{w}_{b}}{\partial x^{2}}-K_{2} \frac{\partial^{2} \ddot{w}_{s}}{\partial x^{2}} \\
& \delta \phi: \frac{\partial Q}{\partial x}-N_{z}=J_{0}\left(\ddot{w}_{b}+\ddot{w}_{s}\right)+K_{0} \ddot{\phi}
\end{aligned}
$$

Equations (15) can be expressed in terms of displacements $\left(u_{0}, w_{b}, w_{s}\right.$ and $\left.\varphi\right)$ by using Eqs. (5), (7), (8) and (12) as follows

$$
\begin{aligned}
& \delta u_{0}: A_{11} d_{11} u_{0}-B_{11} d_{111} w_{b}-B_{11}^{s} d_{111} w_{s}+L d_{1} \phi=I_{0} \ddot{u}_{0}-I_{1} d_{1} \ddot{w}_{b}-J_{1} d_{1} \ddot{w}_{s} \\
& \delta w_{b}: B_{11} d_{111} u_{0}-D_{11} d_{111} w_{b}-D_{11}^{s} d_{1111} w_{s}+L^{a} d_{11} \phi+q=I_{0}\left(\ddot{w}_{b}+\ddot{w}_{s}\right)+ \\
& J_{0} \ddot{\phi}+I_{1} d_{11} \ddot{u}_{0}-I_{2} d_{11} \ddot{w}_{b}-J_{2} d_{11} \ddot{w}_{s} \\
& \delta w_{s}: B_{11}^{s} d_{11} u_{0}-D_{11}^{s} d_{111} w_{b}-H_{11}^{s} d_{1111} w_{s}+A_{55}^{s} d_{11} w_{s}+\left(R+A_{55}^{s}\right) d_{11} \varphi+q= \\
& I_{0}\left(\ddot{w}_{b}+\ddot{w}_{s}\right)+J_{0} \ddot{\varphi}+J_{1} d_{1} \ddot{u}_{0}-J_{2} d_{11} \ddot{w}_{b}-K_{2} d_{11} \ddot{w}_{s} \\
& \delta \varphi: L d_{1} u_{0}-L^{a} d_{11} w_{b}+\left(R+A_{55}^{s}\right) d_{11} w_{s}-A_{55}^{s} d_{11} \varphi+R^{a} \varphi=J_{0}\left(\ddot{w}_{b}+\ddot{w}_{s}\right)+K_{0} \ddot{\varphi}
\end{aligned}
$$

where

$d_{i}=\frac{\partial}{\partial x_{i}}, \quad(i=1)$

where $A_{11}, B_{11}$, etc., are the beam stiffness, defined by

$\left(A_{11}, \quad B_{11}, \quad D_{11}, \quad B_{11}^{s}, \quad D_{11}^{s}, \quad H_{11}^{s}\right)=\int_{-h / 2}^{h / 2} Q_{11}\left(1, z, z^{2}, f, z f, f^{2}\right) d z$ 


$$
\begin{aligned}
& \left(L, L^{a}, R\right)=\int_{-h / 2}^{h / 2} Q_{13}(1, z, f) g^{\prime} d z, \\
& R^{a}=\int_{-h / 2}^{h / 2} Q_{33}\left(g^{\prime}(z)\right)^{2} d z, \\
& A_{55}^{s}=\int_{-h / 2}^{h / 2} Q_{55}(g(z))^{2} d z
\end{aligned}
$$

\section{ANALYTICAL SOLUTIONS}

The equations of motion admit the Navier solutions for simply supported beams. The variables $u_{0}, w_{b}, w_{s}$ and $\varphi$ can be written by assuming the following variations

$$
\left\{\begin{array}{l}
u_{0} \\
w_{b} \\
w_{s} \\
\phi
\end{array}\right\}=\sum_{m=1}^{\infty}\left\{\begin{array}{l}
U_{m} \cos (\lambda x) e^{i \omega t} \\
W_{b m} \sin (\lambda x) e^{i \omega t} \\
W_{s m} \sin (\lambda x) e^{i \omega t} \\
\Phi_{m} \sin (\lambda x) e^{i \omega t}
\end{array}\right\}
$$

where $U_{m}, W_{b m}, W_{s m}$ and $\Phi_{m}$ are arbitrary parameters to be determined, $\omega$ is the Eigen frequency associated with nth Eigenmode, and $\lambda=\mathrm{m} \pi / \mathrm{L}$.

For the beam with two ends simply supported, the boundary conditions are given by

$$
u=w=M_{b}=0 \quad \text { at } \quad x=0, L
$$

Substituting the expansions of $u_{0}, w_{b}, w_{s}$ and $\varphi$ from Eqs. (18) into the equations of motion Eq. (16) . The analytical solutions can be obtained from the following equations

$$
\left(\left[\begin{array}{llll}
S_{11} & S_{12} & S_{13} & S_{14} \\
S_{12} & S_{22} & S_{23} & S_{24} \\
S_{13} & S_{23} & S_{33} & S_{34} \\
S_{14} & S_{24} & S_{34} & S_{44}
\end{array}\right]-\omega^{2}\left[\begin{array}{cccc}
m_{11} & m_{12} & m_{13} & 0 \\
m_{12} & m_{22} & m_{23} & m_{24} \\
m_{13} & m_{23} & m_{33} & m_{34} \\
0 & m_{24} & m_{34} & m_{44}
\end{array}\right]\right)\left\{\begin{array}{l}
U_{m} \\
W_{b m} \\
W_{s m} \\
\Phi_{m}
\end{array}\right\}=\left\{\begin{array}{l}
0 \\
0 \\
0 \\
0
\end{array}\right\}
$$

where

$$
\begin{array}{lll}
s_{11}=\lambda^{2} A_{11}, & s_{12}=-\lambda^{3} B_{11}, & s_{23}=\lambda^{4} D_{11}^{s}, \\
s_{22}=\lambda^{4} D_{11}, & s_{13}=-\lambda^{3} B_{11}^{s}, & s_{24}=\lambda^{2} L^{a}, \\
s_{33}=\lambda^{4} H_{11}^{s}+\lambda^{2} A_{55}^{s} & s_{14}=-\lambda L, & s_{34}=\lambda^{2}\left(A_{55}^{s}+R\right), \\
s_{44}=\lambda^{2} A_{55}^{s}+R^{a} & & \\
m_{11}=I_{0}, & m_{12}=-\lambda I_{1}, & m_{13}=-\lambda J_{1}, \\
m_{22}=I_{0}+\lambda^{2} I_{2}, & m_{23}=I_{0}+\lambda^{2} J_{2}, & m_{24}=m_{34}=J_{0}, \\
m_{33}=I_{0}+\lambda^{2} K_{2}, & m_{44}=K_{0} &
\end{array}
$$

\section{NUMERICAL RESULTS AND DISCUSSION}

\begin{tabular}{|c|c|c|c|c|c|c|c|c|}
\hline \multirow[t]{2}{*}{$\mathrm{L} / \mathrm{h}$} & \multirow[t]{2}{*}{ mode } & \multirow[t]{2}{*}{ theory } & \multicolumn{6}{|c|}{$\mathrm{p}$} \\
\hline & & & 0 & 0.5 & 1 & 2 & 5 & 10 \\
\hline \multirow{9}{*}{5} & \multirow{3}{*}{1} & CBT & 5.395 & 4.593 & 4.148 & 3.779 & 3.595 & 3.492 \\
\hline & & HSDT [12] & 5.153 & 4.411 & 3.990 & 3.626 & 3.400 & 3.281 \\
\hline & & Present & 5.166 & 4.434 & 4.026 & 3.670 & 3.437 & 3.304 \\
\hline & \multirow{3}{*}{2} & CBT & 20.619 & 17.541 & 15.798 & 14.326 & 13.588 & 13.238 \\
\hline & & HSDT [12] & 17.884 & 15.461 & 14.012 & 12.640 & 11.535 & 11.022 \\
\hline & & Present & 17.998 & 15.587 & 14.168 & 12.813 & 11.678 & 11.117 \\
\hline & \multirow{3}{*}{3} & CBT & 43.348 & 36.831 & 33.028 & 29.746 & 28.085 & 27.475 \\
\hline & & HSDT [12] & 34.225 & 29.849 & 27.108 & 24.319 & 21.699 & 20.555 \\
\hline & & Present & 34.556 & 30.168 & 27.459 & 24.676 & 21.985 & 20.753 \\
\hline \multirow{9}{*}{20} & \multirow{3}{*}{1} & CBT & 5.478 & 4.664 & 4.216 & 3.847 & 3.663 & 3.554 \\
\hline & & HSDT [12] & 5.460 & 4.651 & 4.205 & 3.836 & 3.648 & 3.539 \\
\hline & & Present & 5.466 & 4.669 & 4.238 & 3.879 & 3.685 & 3.560 \\
\hline & \multirow{3}{*}{2} & CBT & 21.844 & 18.598 & 16.810 & 15.333 & 14.596 & 14.168 \\
\hline & & HSDT [12] & 21.573 & 18.396 & 16.634 & 15.162 & 14.373 & 13.926 \\
\hline & & Present & 21.603 & 18.475 & 16.769 & 15.336 & 14.519 & 14.013 \\
\hline & \multirow{3}{*}{3} & CBT & 48.899 & 41.633 & 37.617 & 34.295 & 32.636 & 31.688 \\
\hline & & HSDT [12] & 47.594 & 40.653 & 36.769 & 33.468 & 31.572 & 30.534 \\
\hline & & Present & 47.686 & 40.845 & 37.079 & 33.863 & 31.903 & 30.737 \\
\hline
\end{tabular}

In the following computation, the material constituents of the FGM beam are considered to be composed by alumina and aluminum with the material properties as followsAlumina $\left(\mathrm{Al}_{2} \mathrm{O}_{3}\right): \mathrm{E}_{\mathrm{c}}=380 \mathrm{GPa}, \rho_{\mathrm{c}}=3960 \mathrm{~kg} / \mathrm{m}^{3}, v=0.3-$ Aluminum (Al); $E_{m}=70 \mathrm{GPa}, \rho_{\mathrm{m}}=2702 \mathrm{~kg} / \mathrm{m}^{3}, v=0.3$

The dimensionless frequency is defined as

$$
\bar{\omega}=\left(\omega L^{2} / h\right) \sqrt{\rho_{m} / E_{m}}
$$

To check the accuracy of the method used in this investigation, the non-dimensional frequencies $\sigma$ computed by the present theory are compared with those of Ould Larbi et al. [13] and classical beam theory (CBT).

Table 1. First three non-dimensional frequencies $\varpi$ of FG beams

Table 1 presents the first three non-dimensional frequencies of FG beams for different values of power law index $\mathrm{k}$ and span-to-depth ratio $\mathrm{L} / \mathrm{h}$. Results are in good agreements with the published results of Ould Larbi et al.
[13]. The small difference observed between the results obtained by the present theory and Ould Larbi et al. [13] is due to the effect of thickness stretching which is omitted this latter. It can be observed also that there is a remarkable 
difference between the frequencies of CBT and those of shear deformable beam theories for thicker FG beam.

The variation of natural frequencies in terms of the powerlaw index and side-to-thickness ratio is plotted in figure 2 . It can be seen from this figure that the natural frequencies decrease with the increase of the power-law index. It is due to the fact that a higher value of $p$ corresponds to lower value of volume fraction of the ceramic phase, and thus makes the plates become the softer ones.

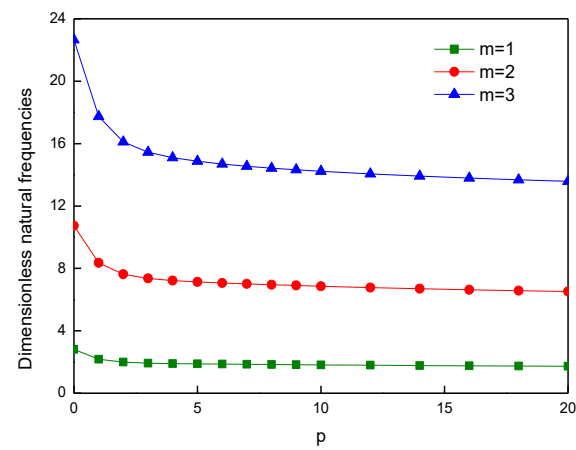

(a)

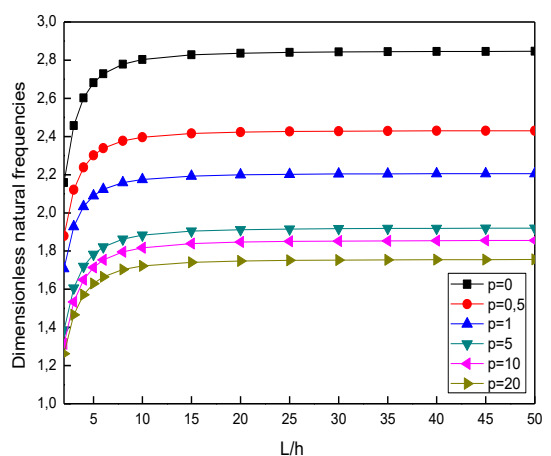

(b)

Figure 2. Effect of the power-law index $\mathrm{p}$ and side-tothickness ratio $\mathrm{L} / \mathrm{h}$ on the natural frequency $\varpi$ of $\mathrm{Al} / \mathrm{Al}_{2} \mathrm{O}_{3}$ beam

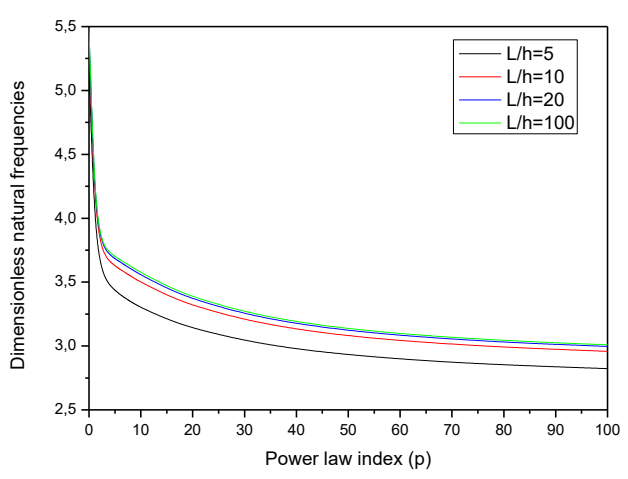

Figure 3. Variation of the non-dimensional fundamental frequency of FG beam with power-law index $\mathrm{p}$ and side-tothickness ratio $\mathrm{L} / \mathrm{h}$.

Figure 3 shows the non-dimensional fundamental natural frequency versus the volume fraction exponent $\mathrm{p}$ for different values of span-to-depth ratio $L / h$. It can be seen from this figure that the full ceramic beams $(p=0)$ lead to a highest frequency. However, the lowest frequency values are predicted for the full metal $(\mathrm{p} \rightarrow \infty)$. This is due to the fact that an increase in the value of the volume fraction exponent results in a decrease of the value of the elasticity modulus. In other words, the beam becomes flexible as the power law exponent increases. Therefore, as also known from mechanical vibrations, natural frequencies decrease as the stiffness of a structure decreases.

\section{CONCLUSION}

The free vibration response of FGM beams is studied based on the higher-order shear deformation theories. This method considers both the shear deformation and thickness stretching effects by a hyperbolic distribution of all displacements through the thickness and without introducing a shear correction factor. The equations of motion for the functionally graded beams are derived from Hamilton's principle. The dimensionless frequencies are presented for the FGM beams with the material properties varying continuously in the thickness direction according to powerlaw form. The effects of the slenderness ratio, the material gradient parameters on the frequencies are examined in detail. The results are validated by comparing them with the results of other researcher. The numerical results show that the effects of the shear deformation on the frequencies tend to be more significant when the beams become shorter (or thicker). Furthermore, for a given length-height ratio, the shear deformation effects are more evident for higher-mode frequencies than for lower-mode frequencies.

\section{ACKNOWLEDGMENT}

The authors are thankful to all members of Material and Hydrology Laboratory from Djillali Liabes University for their support.

\section{REFERENCES}

[1] Şimşek M., Kocatürk T. (2009). Free and forced vibration of a functionally graded beam subjected to a concentrated moving harmonic load, Composite Structures, Vol. 90, No. 4, pp. 465-473. DOI: 10.1016/j.compstruct.2009.04.024

[2] Yang J., Chen Y. (2008). Free vibration and buckling analysis of functionally graded beams with edge cracks, Composite Structures, Vol. 83, No. 1, pp. 4860. DOI: $10.1016 /$ j.compstruct.2007.03.006

[3] Li S.R., Su H.D., Cheng C.J. (2009). Free vibration of functionally graded material beams with surfacebonded piezoelectric layers in thermal environment, Applied Mathematics and Mechanics (English Edition), Vol. 30, No. 8, pp. 969-982. DOI: $\frac{10.1007 / \mathrm{s} 10483-009-0803-7}{\text { X.F. }}$

[4] Li X.F. (2008). A unified approach for analyzing static and dynamic behaviors of functionally graded Timoshenko and Euler-Bernoulli beams, Journal of Sound and Vibration, Vol. 318, No. 4, pp. 1210-1229. DOI: $\underline{10.1016 / j . j s v .2008 .04 .056}$

[5] Huang Y., Li X.F.A. (2010). New approach for free vibration of axially functionally graded beams with non-uniform cross-section, Journal of Sound and 
Vibration, Vol. 329, No. 11, pp. 2291-2303. DOI: 10.1016/j.jsv.2009.12.029

[6] Bouremana M., Houari M.S.A., Tounsi A., Kaci A., Bedia E.A.A. (2013). A new first shear deformation beam theory based on neutral surface position for functionally graded beams, Steel and Composite Structures, Vol. 15, No. 5, pp. 467-479. DOI: 10.12989/scs.2013.15.5.467

[7] Aydogdu M., Tashkin V. (2007). Free vibration analysis of functionally graded beams with simply supported edges, Materials and Design, Vol. 28, No. 5, pp. 1651-1656. DOI: 10.1016/j.matdes.2006.02.007

[8] Şimşek M. (2010). Fundamental frequency analysis of functionally graded beams by using different higher order beam theories, Nuclear Engineering and Design, Vol. 240, No. 4, pp. 697-705. DOI: 10.1016/j.nucengdes.2009.12.013

[9] Mahi A., Bedia E.A.A., Tounsi A., Mechab I. (2010). An analytical method for temperature dependent free vibration analysis of functionally graded beams with general boundary conditions, Composite Structures, Vol. 92, pp. 1877-1887. DOI: 10.1016/i.compstruct.2010.01.010

[10] Ahmed B., Fouad B., Djalil B.A., Mohamed B.B., Abdelouahed T., Bedia E.A. (2016). The thermal study of wave propagation in functionally graded material plates (FGM) based on neutral surface position, Mathematical Modelling of Engineering Problems, Vol. 3, No. 4, pp. 202-205. DOI: $\underline{10.18280 / \mathrm{mmep} .030410}$

[11] Bourada M., Kaci A., Houari M.S.A., Tounsi A. (2015). A new simple shear and normal deformations theory for functionally graded beams, Steel and Composite Structures, Vol. 18, No. 2, pp. 409-423. DOI: $10.12989 / \mathrm{scs} .2015 .18 .2 .409$

[12] Zenkour A.M. (2013). A simple four-unknown refined theory for bending analysis of functionally graded plates, Appl. Math. Model., Vol. 37, No. 20-21, pp. 9041-9051. DOI: 10.1016/j.apm.2013.04.022

[13] Ould L.L., Kaci A., Houari M.S.A., Tounsi A. (2013) An efficient shear deformation beam theory based on neutral surface position for bending and free vibration of functionally graded beams, Mech. Based Des. Struct. Mach., Vol. 41, No. 4, pp. 421-433. DOI: $\underline{10.1080 / 15397734.2013 .763713}$

\section{NOMENCLATURE}

K

$\mathrm{U}$

w

\section{Greek symbols}

$\tau$

\section{Superscripts}

i

\section{Subscripts}

$\begin{array}{ll}\mathrm{b} & \text { Bending } \\ \mathrm{c} & \text { ceramic } \\ \mathrm{n} & \text { mode } \\ \mathrm{m} & \text { metal } \\ \mathrm{s} & \text { Shear }\end{array}$

variation

temps

Shear
Width, mm

Stiffness coefficient Young's modulus, N.m-2 Thickness, mm inertia term kinetic energy, J Length, mm Material property external forces, $\mathrm{kN}$ Axial displacement, $\mathrm{mm}$ strain energy, J work done, J

Transverse displacement, $\mathrm{mm}$

Normal strain

Shear strain, rad

Normal stress, N.m-2

Shear stress, N.m-2

Frequencie $\mathrm{Hz}$

Mass density kg. m-3

Coefficient de Poisson

differentiation

Laplacian operator

Power law index

fluid (pure water)

differentiation with respect to the time variable 